\title{
Sedation with ketamine and fentanyl combination improves patient outcomes in intensive care units
}

\author{
Sarah Vitug ${ }^{1} \cdot$ Vikas Ravi $^{2} \cdot$ Duraiyah Thangathurai $^{3}$
}

Accepted: 6 April 2020 / Published online: 10 July 2020

(C) Springer Nature Switzerland AG 2020

\begin{abstract}
Psychological manifestations such as depression and suicidal ideation are commonly caused by poorly controlled pain, anxiety, and sleep deprivation in intensive care unit (ICU) patients. We are concerned that previous analgesic and sedative techniques administered as single-medication approaches are outdated and inadequate. It is imperative that ICU practitioners are knowledgeable in multimodal approaches to pain and sedation in high acuity settings. We have shown that appropriate combinations of ketamine and fentanyl are effective, and if further supplementation is needed, we utilize additional pharmacological agents in low doses and regional techniques that ultimately lower the overall opioid consumption. We acknowledge that a variety of medication supplementations tailored to the patient's clinical needs and nature of surgery improves a patient's outcome in ICU and overall quality of life.
\end{abstract}

Keywords Ketamine $\cdot$ Fentanyl $\cdot$ Psychological effects $\cdot$ ICU $\cdot$ Patient outcome

Pain, anxiety, and sleep deprivation are commonly experienced by intensive care unit (ICU) patients. Often, these issues may result in psychological manifestations such as severe depression, demoralization, hopelessness, delirium, psychosis, delusions, PTSD, and, occasionally, suicidal ideations. In these scenarios, patients lose the will to live, as severe hopelessness and learned helplessness may cloud decision-making which may result in patient request for termination of care.

The neuroendocrine stress responses often associated with noxious conditions can produce deleterious hemodynamic,

This article is part of the Topical Collection on Medicine
Sarah Vitug
sarah.vitug@uq.net.au
Vikas Ravi
vikasravi@oakland.edu
Duraiyah Thangathurai
thangath@med.usc.edu

1 Ochsner Clinical School, University of Queensland, 1514 Jefferson Highway, New Orleans, LA 70121, USA

2 Oakland University William Beaumont School of Medicine, 586 Pioneer Drive, Rochester, MI 48309, USA

3 Department of Anesthesiology, Keck Medical Center of the University of Southern California, 1500 San Pablo St, Rm. 808TB/ 8E, Los Angeles, CA 90033, USA metabolic, nutritional, and immunologic changes. Prevention or timely treatment of symptoms can reduce the need for large doses of sedatives. However, irritation by the endotracheal tube may result in coughing and fighting the ventilator, which necessitates large doses of sedatives. This further complicates the management of patients on ventilatory support. Asynchrony between spontaneous ventilatory efforts and machine-delivered breaths predisposes to pulmonary barotrauma, interferes with alveolar gas exchange, and increases the work of breathing [1]. Inappropriate hyperventilation is a common occurrence and may lead to hypocapnia, respiratory alkalosis, and hemodynamic disturbances. For these reasons, sedation is mandated, which also provides amnesia. Neuromuscular paralysis may be used to decrease patient resistance to ventilatory support; however, side effects such as prolonged muscle weakness and myopathic changes are associated with these drugs.

Earlier sedation practices relied primarily on intermittent intravenous narcotics such as morphine sulfate, meperidine, or methadone [2]. Benzodiazepines such as diazepam, lorazepam, and midazolam, and barbiturates such as phenobarbital and pentobarbital were given when amnesia or hypnosis was required. These drugs are known to cause addiction, potentially leading to increased tolerance and functional changes within the brain. Long-acting sedatives and narcotics often cause respiratory and circulatory depression, which may prolong the stay on respirators. Opioid sedatives can also decrease 
gastrointestinal function and increase the risk of aspiration pneumonia, by slowing motility and prolonging ileus.

For the last 25 years, we have adopted a multimodal approach for pain and sedation, such as ketamine and fentanyl combinations for many of our postoperative ICU patients. Of note, the majority of our patient population have undergone major oncologic surgeries at the Norris Cancer Institute/USC. A multimodal approach minimizes the requirement of individual medications and their potential side effects. In patients who are resistant to sedation, we add medication in addition to ketamine and fentanyl, such as low-dose propofol, midazolam, or dexmedetomidine [3]. If analgesic requirement is high, we supplement with IV infusions of acetaminophen.

Ketamine, in particular, has many beneficial effects such as hemodynamic stability, bronchodilation, and minimal respiratory depression. Ketamine also has many powerful analgesic and amnesic properties [4]. Ketamine is used in acute and resistant-depressive states, which is common in the ICU [5]. Although the rate of hallucinations and nightmares is less than $5 \%$, these psychotomimetic reactions are attenuated when ketamine is combined with a benzodiazepine or opioid. A concomitant infusion of an opioid or ketamine augments the sedation, provides analgesia, and reduces drug requirements. Adding fentanyl to ketamine as an appropriate combination can provide excellent analgesia and reduce further sedative or analgesic requirements. We have been using a ketamine and fentanyl combination in surgical oncology ICU for over 30 years with excellent results, including early extubation, early return of gastrointestinal function, fewer hemodynamic/ respiratory complications, minimal psychological issues including depression and psychosis, shorter ICU stays, and overall decreased morbidity and mortality.

The purpose of our multimodal approach to pain and sedation in the postoperative ICU setting is to improve the quality of care for our patients by minimizing undesired effects associated with analgesic agents. In standard practice, ketamine is commonly used in larger doses for induction and anesthesia maintenance. Intraoperatively, higher doses of ketamine are associated with hypertension, tachycardia, and arrhythmias. Postoperatively, hallucinations and psychotic states are occasionally associated with ketamine. Ketamine use may result in medication-related psychotic states in patients with a history of psychoses such as schizophrenia or similar disorders, substance abuse/withdrawal, or medication-induced or metabolic-related causes. Thus, we take extra precautions in patients with relevant psychiatric or medical histories. We found that by using sub-anesthetic doses of ketamine in combination with either low-dose fentanyl or midazolam, the incidence of psychotic symptoms is minimized. Occasionally, we supplement ketamine with low-dose dexmedetomidine infusion or quetiapine (Seroquel) in those who develop persistent psychotic symptoms. We acknowledge that even lowdose ketamine and supplementary analgesic/sedative agents can precipitate unwanted medication-induced psychotic symptoms and other side effects; thus, continued close monitoring of postoperative ICU patients under this multimodal regimen is advised.

Authors' Contributions All authors dutifully fulfill the following criteria: - Substantial contributions to the conception or design of the work or the acquisition, analysis, or interpretation of data for the work

- Drafting the work or revising it critically for important intellectual content

- Final approval of the version to be published

- Agreement to be accountable for all aspects of the work in ensuring that questions related to the accuracy or integrity of any part of the work are appropriately investigated and resolved

\section{Compliance with Ethical Standards}

Conflict of Interest The authors declare that they have no conflict of interest.

\section{References}

1. Mikhail MS, Thangathurai D. Sedating patients in intensive care units. West J Med. 1992;157(5):566.

2. Aitkenhead AR. Analgesia and sedation in intensive care. $\mathrm{Br} \mathbf{J}$ Anaesth. 1989;63(2):196-206.

3. Shehabi Y, Howe BD, Bellomo R, Arabi YM, Bailey M, Bass FE, et al. Early sedation with dexmedetomidine in critically ill patients. $\mathrm{N}$ Engl J Med. 2019;380(26):2506-17.

4. Tobias JD, Martin LD, Wetzel RC. Ketamine by continuous infusion for sedation in the pediatric intensive care unit. Crit Care Med. 1990;18(8):819-21.

5. Hirota K, Lambert DG. Ketamine and depression. Br J Anaesth. 2018;121(6):1198-202.

Publisher's Note Springer Nature remains neutral with regard to jurisdictional claims in published maps and institutional affiliations. 